\title{
First Principles Study on the Two-dimensional Germanium Arsenide Containing Vacancy
}

\author{
Yi Wei ${ }^{1,}$, , Liang Fang ${ }^{1}$, Xianyu Tong ${ }^{1}$, Rulin Liu ${ }^{2}$ \\ ${ }^{1}$ Institute for Quantum Information \& State Key Laboratory of High Performance Computing, National University of Defense Technology, \\ Changsha, China \\ ${ }^{2}$ College of Computer, National University of Defense Technology, Changsha, China
}

\section{Email address:}

hiweiyi@163.com (Yi Wei)

${ }^{*}$ Corresponding author

\section{To cite this article:}

Yi Wei, Liang Fang, Xianyu Tong, Rulin Liu. First Principles Study on the Two-Dimensional Germanium Arsenide Containing Vacancy. Science Discovery. Vol. 7, No. 4, 2019, pp. 188-193. doi: 10.11648/j.sd.20190704.11

Received: May 29, 2019; Accepted: July 17, 2019; Published: July 19, 2019

\begin{abstract}
Two-dimensional layered materials exhibit exotic optical, electrical and thermoelectric properties, which have drawn worldwide attention in the past decade. As a novel kind of two-dimensional semiconductor, monolayer Germanium Arsenide (GeAs) can be exfoliated from bulk solid and it owns excellent dynamical and thermal stability and tunable bandgap. Based on the first principles calculations, we investigate the geometric structure, electrical and magnetic properties of the pristine, Germanium vacancy $\left(\mathrm{V}_{\mathrm{Ge}}\right)$ defected and Arsenide vacancy $\left(\mathrm{V}_{\mathrm{As}}\right)$ defected monolayer GeAs. In agreement with the experimental results, the pristine monolayer GeAs is a direct bandgap semiconductor with a forbidden band width of $1.48 \mathrm{eV}$. By applying a full spin-polarized description to the system, we find that pristine monolayer GeAs is non-magnetic. Introduction of an atomic vacancy defect in the unit cell of monolayer GeAs can lead to six unequal structures. The most stable structure of monolayer GeAs with $\mathrm{V}_{\mathrm{Ge}}$ is also a direct bandgap semiconductor with a forbidden band width of $0.17 \mathrm{eV}$ and is non-magnetic. The most stable structure of monolayer GeAs with $\mathrm{V}_{\mathrm{As}}$ can generate a magnetic moment of $0.4 \mu_{\mathrm{B}}$, and it has desired half-metallic behavior. These intriguing results indicate that both pristine and intrinsic vacancy defected monolayer GeAs are promising candidates for two-dimensional optoelectronics and spintronic devices with high performance.
\end{abstract}

Keywords: First Principles, Two-dimensional Material, Germanium Arsenide, Direct Bandgap Semiconductors, Vacancy Defects, Half-mental

\section{含缺陷单层砷化锗的第一性原理研究}

\author{
魏伊 ${ }^{1^{*}}$, 方粮 ${ }^{1}$ ，童仙雨 ${ }^{1}$, 刘汝霖 ${ }^{2}$ \\ ${ }^{1}$ 国防科技大学量子信息研究所\&高性能计算国家重点实验室, 长沙, 中国 \\ ${ }^{2}$ 国防科技大学计算机学院, 长沙, 中国
}

\section{邮箱}

hiweiyi@163.com（魏伊）

摘要: 二维材料因其独特的光学和电学特性近年来受到广泛关注。单层砷化锗 $(\mathrm{GeAs})$ 是一种新型的二维半导体材料, 具有良好的动力学和热力学稳定性和可调带隙, 并且可以从块体材料中剥离。本文通过第一性原理计算方法, 研究了 本征及具有锗空位缺陷 $\left(\mathrm{V}_{\mathrm{Ge}}\right)$ 和砷空位缺陷 $\left(\mathrm{V}_{\mathrm{As}}\right)$ 的单层 $\mathrm{GeAs}$ 的几何结构、电学和磁学性质。研究发现, 单层 $\mathrm{Ge} A s$ 是一 种具有 $1.48 \mathrm{eV}$ 带隙的直接带隙半导体, 与实验结果相同。对系统添加全自旋极化, 发现本征单层GeAs无磁性。在单层 GeAs单胞中引入单一本征空位缺陷可以产生六种不等价体系, 其中最稳定的锗空位缺陷体系是具有 $0.17 \mathrm{eV}$ 带隙的直接 
带隙半导体且无磁性; 最稳定的砷空位缺陷体系可产生 $0.4 \mu_{\mathrm{B}}$ 的磁矩并呈现出半金属特性。研究结果表明本征和含空位 缺陷的单层GeAs材料都是二维高性能光电子器件和自旋电子器件的候选材料。

关键词：第一性原理，二维材料，砷化锗，直接带隙半导体，空位缺陷，半金属

\section{1. 引言}

IV 族单质元素组成的二维材料如石墨烯、硅烯、锗烯 和锡烯等, 因其具有独特的结构和优良的力学、热学和光 学性能而受到广泛关注[1-7], 但其均缺乏本征带隙, 限制 了其在发光二极管、太阳能电池以及逻辑电路等方面的应 用。而 $\mathrm{V}$ 族单质元素组成的二维材料如黑磷, 虽然具有连 续可调带隙, 但黑磷在空气不稳定, 限制了其在未来的应 用 $[8,9]$ 。将IV族元素与 $V$ 族元素相结合, 可以得到一种集 两族元素优点于一身的二维材料。这种 IV $-\mathrm{V}$ 族二维材料, 如砷化锗 $(\mathrm{GeAs})$, 磷化锗 $(\mathrm{GeP})$, 砷化硅 $(\mathrm{SiAs})$, 磷化硅 $(\mathrm{SiP})$ 等均是一种新型的具有低对称性的二维材料 [10-15], 其中 二维GeAs材料具有良好的动力学和热力学稳定性、显著 的各向异性和较小的载流子有效质量等诸多优异的性能, 近年来引起了越来越多的关注。

2017年, Zhou等[13]计算得到单层GeAs具有良好的动 力学和热力学稳定性, 层间结合能很小, 较容易从自然界 中存在的大块固体中剥落, 且具有相对较小且各向异性的 载流子有效质量, 表明其可实现快速的电输运; 2018年, Jung等[16]采用液相剥离法制备了单层GeAs纳米片, 发现单 层GeAs 具有良好的稳定性, 能产生较大且稳定的光电流, 且可见光照射下显示出良好的光电化学分解水的性能, 表明 了其在高性能光电纳米器件中的潜在应用; 同年, Yang等[17] 实验测得单层GeAsi移率的各向异性比为 4.6 , 高于黑磷等
其他二维各向异性材料;同年, Guo等[18]制备了少数层 $\mathrm{GeAs}$ 场效应晶体管, 其在室温下为空穴导电型半导体, 载流子迁 移率接近 $100 \mathrm{~cm}^{2} \mathrm{~V}^{-1} \mathrm{~s}^{-1}$ 且开关电流比超过了 $10^{5}$, 与最先进 的过渡金属硫化物晶体管性能相当, 且其可以对中红外辐射 进行快速强烈的光响应。这些结果都为单分子或少数分子层 的GeAs材料的合成以及它们在光催化、纳米电子和光电子 器件等方面的应用提供了有价值的指导。

由于实验条件有限, 在制备二维材料时不可避免的会 引入各种缺陷。现实验与计算方面大量的研究工作都比较 关注缺陷对体系的稳定性、微观几何结构、电子结构和磁 性等基本性质的影响 [19-21], 其中空位缺陷的存在会对材 料的电子结构和磁性带来一些非常有意义的变化。目前还 没有对空位缺陷掺杂对单层GeAs性能影响的研究, 所以 本文通过第一性原理计算并分析了本征及含空位缺陷的 单层GeAs的稳定性、几何结构、电学和磁学性质。

\section{2. 模型与计算方法}

\section{1. 构建模型}

理想的 GeAs 晶体结构如图 1 所示, 空间群为 $\mathrm{C} 2 / \mathrm{m}(\mathrm{No} .12)[12]$ 。每层GeAs都以As原子终止并通过弱范 德华力与其他层相互作用, 层厚度为 $6.6 \AA$ 。

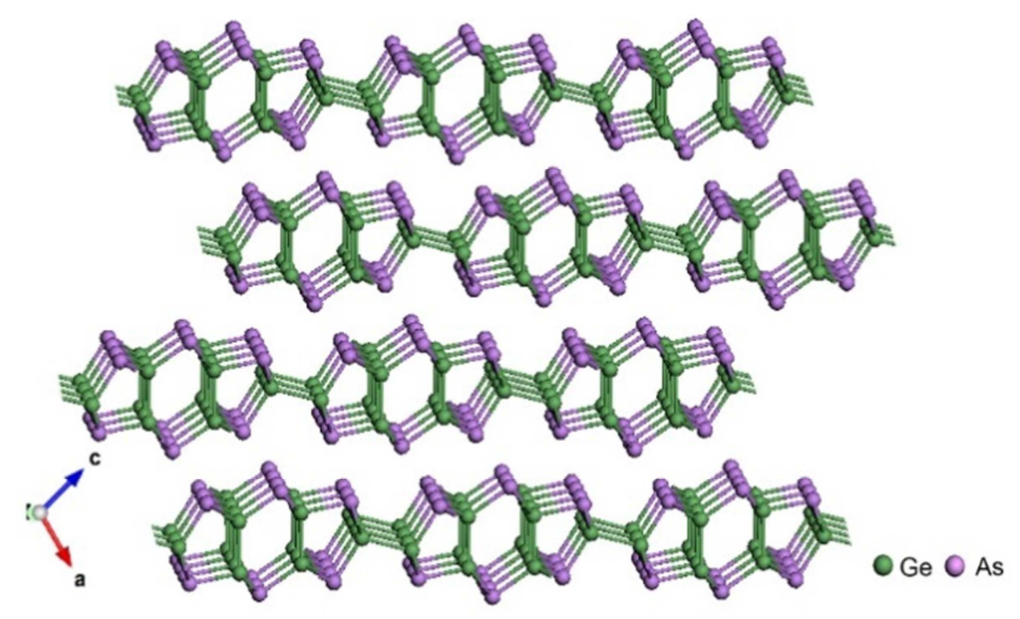

图1 GeAs晶体层状结构图。

图2为单层 $\mathrm{GeAs}$ 结构的俯视图和侧视图, 单胞由图2(a) 中黑色矩形标记。俯视图可以看出单层 GeAs单胞包含 12 个 $\mathrm{Ge}$ 原子和 12 个 As 原子。侧视图中可以看出结构由五边 形和六边形环组成。在每一层中, Ge-Ge原子对被6个 $\mathrm{As}$ 原子包围, 每个As原子与三个 $\mathrm{Ge}$ 原子相协调, 每个 $\mathrm{Ge}$ 原 子与三个As和另一个 Ge原子相协调, 其中两种 $\mathrm{Ge}-\mathrm{Ge}$ 键分
别平行和垂直于层平面, 说明了 $\mathrm{GeAs}$ 晶体结构的各向异 性。计算体系选取了单层GeAs结构的 $(-2,0,1)$ 面, 为了避 免c轴方向的层间相互作用, 在 $\mathrm{c}$ 方向上加上 $15 \AA$ 的真空层。 值得注意的是, Ge1、Ge2、Ge3分别和 Ge4、Ge5、Ge6, 和 $\mathrm{Ge} 7 、 \mathrm{Ge} 8 、 \mathrm{Ge} 9$, 和 $\mathrm{Ge} 10 、 \mathrm{Ge} 11 、 \mathrm{Ge} 12$ 位置状态是一 样的, As原子同理, 所以单层GeAs中锗空位缺陷和砷空 
位缺陷分别有三种存在方式, 分别标记为 $\mathrm{V}_{\mathrm{Ge} 1}$, $\mathrm{V}_{\mathrm{Ge} 2}, \mathrm{~V}_{\mathrm{Ge} 3}, \mathrm{~V}_{\mathrm{As} 1}, \mathrm{~V}_{\mathrm{As} 2}, \mathrm{~V}_{\mathrm{As} 3}$, 空缺原子分别对应于图2(b)中 $\mathrm{Ge} 1, \mathrm{Ge} 2, \mathrm{Ge} 3, \mathrm{As} 1, \mathrm{As} 2, \mathrm{As} 3$ 。研究单层GeAs空位缺 陷性质时, 为了避免相邻空位缺陷掺杂的相互影响, 采用 了 $1 \times 6$ 的超胞结构, 此时掺杂一个原子的空位缺陷时, 缺 陷浓度为 $0.69 \%$ 。
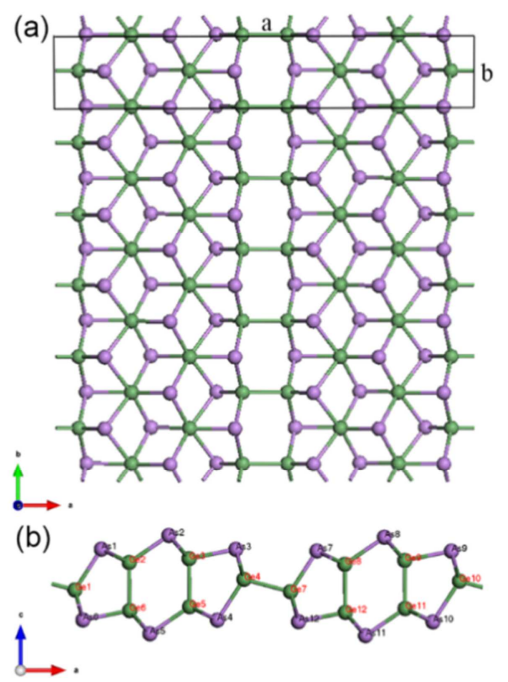

图2 单层GeAs结构图 (a)单层 $\mathrm{GeAs}$ 的 $1 \times 6$ 超胞俯视图; (b)单层 $\mathrm{GeAs}$ 的 $1 \times 6$ 超胞侧视图。

\section{2. 计算方法}

本文采用基于密度泛函理论的第一性原理噟势法, 利 用QUANTUM ESPRESSO(QE)软件进行计算[22,23]。计算 中, 交换关联能采用了广义梯度近似(GGA)[24]中的 Perdew-Burke-Ernzerhof for solid(PBEsol)泛函[25]。经过收 玫测试, 波函数截断能设定为 $48 \mathrm{Ry}$, 电子密度截断能设定 为420Ry, 自洽循环迭代收敛阈值为 $1 \times 10^{-6} \mathrm{eV} / \mathrm{atom}$ 。在 晶格驰豫过程中, 固定 $\mathrm{c}$ 轴长度, 单个原子能量收玫精度 为 $5 \times 10^{-5} \mathrm{eV}$, 原子受力最大值为 $0.01 \mathrm{eV} / \AA$ 。 $\mathrm{K}$ 点采用 Monkhorst-Pack(MP)取样方法[26], 研究本征单层GeAs电 子性质时, $\mathrm{K}$ 点取样为 $3 \times 18 \times 1$, 研究单层 $\mathrm{GeAs}$ 空位缺陷性 质时, 由于晶胞足够大, 结构优化时 $\mathrm{K}$ 点取样为gamma点, 计算能带及态密度时采用 $2 \times 2 \times 1$ 。能带计算时高对称点的 选取路径为 $\mathrm{G}(0.0,0.0,0.0)-\mathrm{Y}(0.0,0.5,0.0)-\mathrm{S}(0.5,0.5$, $0)-\mathrm{X}(0.0,0.5,0.0)-\mathrm{G}(0.0,0.0,0.0)$ 。

\section{3. 结果与讨论}

\section{1. 单层GeAs的电子结构}

首先对单层GeAs单胞进行结构优化, 经过优化后得 到其晶格常数为 $\mathrm{a}=21.5253 \AA, b=3.7047 \AA, c=21.5023 \AA$ 。 图3给出了单层 $\mathrm{GeAs}$ 的能带结构, 从图中可以看出单层 GeAs 是一种直接带隙半导体, 导带底和价带顶均在 $\mathrm{G}$ 点, 禁带宽度为 $1.48 \mathrm{eV}$, 与Cheng等[27]计算得到的单层 $\mathrm{GeAs}$ 是带隙为 $1.42 \mathrm{eV}$ 的直接带隙半导体结果相近。当电子在跃 迁过程中, 电子波矢不变, 即动量不变; 同样, 如果导带
电子下落到价带时, 也可以保持动量不变, 不需要声子来 接受或提供动量, 这种直接复合可以把能量几乎全部以光 的形式放出, 发光效率高, 可用于制备发光器件[28]。

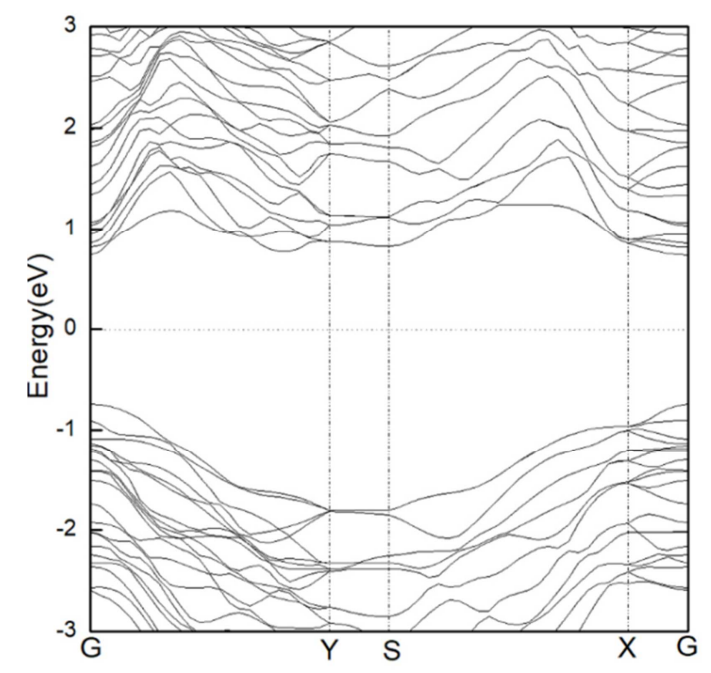

图3 单层GeAs的能带结构。

图4给出了单层GeAs的分波态密度(PDOS), 从图中可 以看出单层 $\mathrm{GeAs}$ 在费米能级处没有电子态填充, 且自旋 向上和自旋向下的分波态密度完全对称, 所以体系为半导 体材料且没有磁性。价带顶主要由As-p, Ge-s和Ge-p组成, 且其对成键的贡献大致相等, 导带底主要由As-p和Ge-s组 成, 与Cheng等[27]计算结果一致。

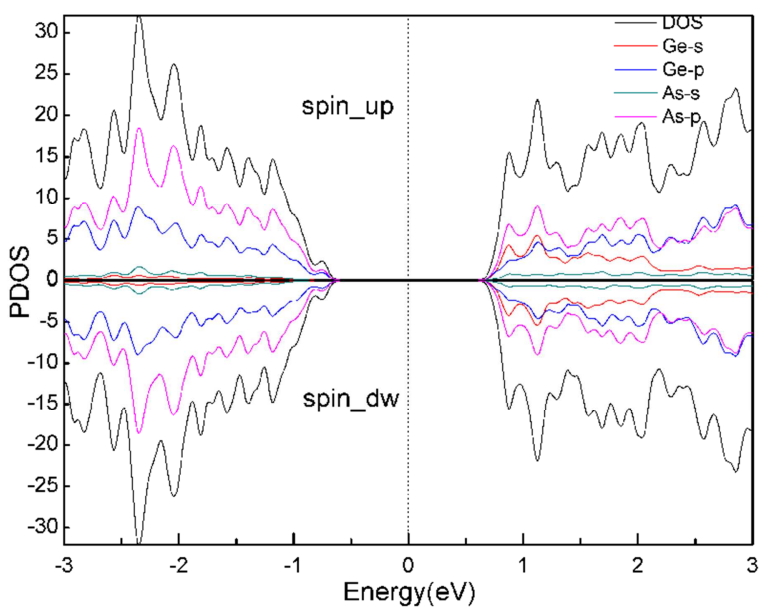

图4 单层GeAs的分波态密度。

\section{2. 单层GeAs掺杂体系的晶体结构}

单层 $\mathrm{GeAs}$ 中 $\mathrm{V}_{\mathrm{Ge}}$ 和 $\mathrm{V}_{\mathrm{As}}$ 分别有三种存在方式。首先对这 六种掺杂体系进行结构优化并计算其结合能 $E_{b}$, 计算方法 由公式(1)表示:

$$
E_{b}=\left[E_{d}-n_{\mathrm{Ge}} E_{i}(\mathrm{Ge})-n_{\mathrm{As}} E_{i}(\mathrm{As})\right] /\left(n_{\mathrm{Ge}}+n_{\mathrm{As}}\right)
$$

式中 $E_{d}$ 为计算中所采用的掺杂体系优化后的总能量, $E_{i}(\mathrm{Ge})$ 和 $E_{i}(\mathrm{As})$ 分别为单个孤立 $\mathrm{Ge}$ 和 $\mathrm{As}$ 的能量, $n_{\mathrm{Ge}}$ 和 $n_{\mathrm{As}}$ 
分别为计算所采用的超胞中所包含 $\mathrm{Ge}$ 和 $\mathrm{As}$ 的个数。计算 结果如表1所示。

表1 掺杂体系结合能。

\begin{tabular}{lll}
\hline 掺杂类型 & 空位原子 & $\boldsymbol{E}_{\boldsymbol{b}} / \mathbf{e V}$ \\
\hline $\mathrm{V}_{\mathrm{Ge} 1}$ & $\mathrm{Ge} 1$ & -5.1142 \\
$\mathrm{~V}_{\mathrm{Ge} 2}$ & $\mathrm{Ge} 2$ & -5.1218 \\
$\mathrm{~V}_{\mathrm{Ge} 3}$ & $\mathrm{Ge} 3$ & -5.1165 \\
$\mathrm{~V}_{\mathrm{As} 1}$ & $\mathrm{As} 1$ & -5.1175 \\
$\mathrm{~V}_{\mathrm{As} 2}$ & $\mathrm{As} 2$ & -5.1205 \\
$\mathrm{~V}_{\mathrm{As} 3}$ & As3 & -5.1197 \\
\hline
\end{tabular}

结果表明, 六种掺杂体系的结合能均为负值, 说明掺 杂体系结构稳定。其中 $V_{\mathrm{Ge} 2}$ 和 $\mathrm{V}_{\mathrm{As} 2}$ 结合能相对较低, 说明 这两种体系更稳定, 也更容易形成。所以接下来的讨论只 考虑 $\mathrm{V}_{\mathrm{Ge} 2}$ 和 $\mathrm{V}_{\mathrm{As} 2}$ 掺杂。

$\mathrm{V}_{\mathrm{Ge} 2}$ 和 $\mathrm{V}_{\mathrm{As} 2}$ 掺杂体系结构优化后晶格参数如表 2 所示。 表中可以看出 $\mathrm{V}_{\mathrm{Ge} 2}$ 和 $\mathrm{V}_{\mathrm{As} 2}$ 掺杂后晶格的长和宽均略有减小, 总体积分别减小为原来的 $99.03 \%$ 和 $98.93 \%$, 说明空位缺陷 掺杂后晶体略有减小，但是总体变化不大。

表2 掺杂体系晶格常数。

\begin{tabular}{lll}
\hline \multirow{2}{*}{ 掺杂类型 } & 晶格常数 & \\
\cline { 2 - 3 } & $\mathbf{a} / \boldsymbol{\AA}$ & $\mathbf{b} / \AA$ \\
\hline 未掺杂 & 21.5253 & 22.2282 \\
$\mathrm{~V}_{\mathrm{Ge} 2}$ & 21.3326 & 22.2110 \\
$\mathrm{~V}_{\mathrm{As} 2}$ & 21.4184 & 22.0995 \\
\hline
\end{tabular}

掺杂晶体结构优化后晶体结构如图5所示, 红色圆圈 中心为空位缺陷。图中可以看出 $\mathrm{V}_{\mathrm{Ge} 2}$ 和 $\mathrm{V}_{\mathrm{As} 2}$ 附近原子均向 缺陷处靠拢, 而距空位缺陷较远处的原子位置并无太大变 化, 晶体整体形变不大, 与之前结论一致。图 $5(\mathrm{a})$ 是 $\mathrm{V}_{\mathrm{Ge}}$ 掺 杂体系, 可以看出 $\mathrm{V}_{\mathrm{Ge} 2}$ 附近的三个As原子明显向缺陷处靠 拢, As1-As2 键长由 $4.055 \AA$ 减小为 $2.757 \AA$, 两单胞间的 As1-As1键长由 $3.833 \AA$ 减小为 $3.562 \AA$ 。图 $5(\mathrm{~b})$ 是 $\mathrm{V}_{\mathrm{As} 2}$ 掺杂体 系, 可以看出 $\mathrm{V}_{\mathrm{As} 2}$ 附近的三个 $\mathrm{Ge}$ 原子明显向缺陷处靠拢, $\mathrm{Ge} 2-\mathrm{Ge} 3$ 键长由 $3.594 \AA$ 减小为 $3.151 \AA$, 两单胞间的 $\mathrm{Ge} 3-\mathrm{Ge} 3$ 键长由 $3.833 \AA$ 减小为 $2.902 \AA$ 。 (a)

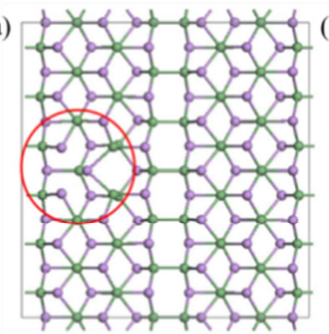

(c)

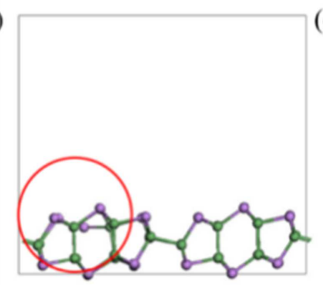

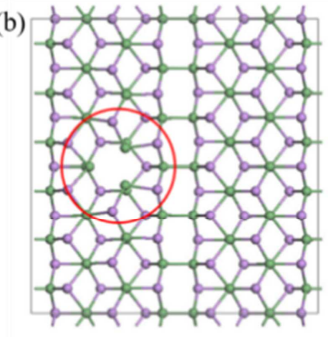

(d)

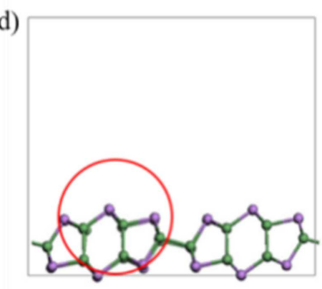

图5 掺杂体系晶格结构(a) $\mathrm{V}_{\mathrm{Ge} 2}$ 体系俯视图; (b) $\mathrm{V}_{\mathrm{As} 2}$ 体系俯视图; (c) $\mathrm{V}_{\mathrm{Ge} 2}$ 体 系侧视图; (d) $\mathrm{V}_{\mathrm{As} 2}$ 体系侧视图。

\section{3. 单层GeAs掺杂体系的电子结构}

$\mathrm{V}_{\mathrm{Ge} 2}$ 和 $\mathrm{V}_{\mathrm{As} 2}$ 掺杂后能带结构如图6所示, 虚线表示费米 能级。图6(a) 是 $\mathrm{V}_{\mathrm{Ge} 2}$ 掺杂体系的能带图, 可以看出 $\mathrm{V}_{\mathrm{Ge} 2}$ 掺 杂体系费米能级上方和下方均出现了一条局域能级, 使体 带隙减小为 $0.17 \mathrm{eV}$, 但仍是一种直接带隙半导体。图6(b) 是 $\mathrm{V}_{\mathrm{As} 2}$ 掺杂体系, 可以看出其费米能级处产生了带隙。
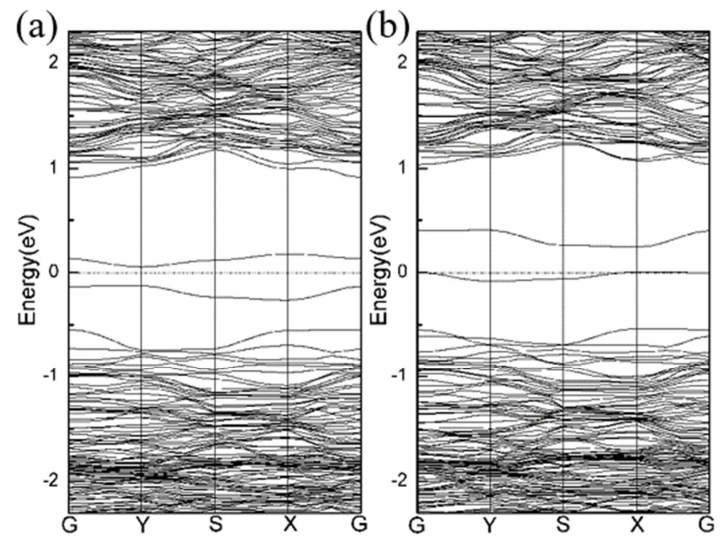

图6 掺杂体系能带结构(a) $\mathrm{V}_{\mathrm{Ge} 2}$ 体系; (b) $\mathrm{V}_{\mathrm{As} 2}$ 体系。

图7为 $\mathrm{V}_{\mathrm{Ge} 2}$ 和 $\mathrm{V}_{\mathrm{As} 2}$ 体系的总态密度。图7(a) 是 $\mathrm{V}_{\mathrm{Ge} 2}$ 体系 态密度图, 可以看出 $\mathrm{V}_{\mathrm{Ge} 2}$ 体系禁带处均生成明显的杂质能 级, 自旋向上和自旋向下的态密度完全对称, 因此体系不 具有磁性。图7(b) 是 $\mathrm{V}_{\mathrm{As} 2}$ 体系态密度图, 可以看出自旋向 上和自旋向下的态密度图出现不对称的现象: 自旋向上和 自旋向下的轨道发生䢃裂, 导致自旋向下的态密度穿越费 米能级, 表现为金属性, 而自旋向上的态密度不穿越费米 能级, 依然为半导体。由于这种上旋和下旋轨道的金属性 和非金属性差别, 导致材料具有独特的性能, 这种材料称 为半金属材料(half-metal), 它有可能在新一代微电子设备 中发挥重要作用, 并为极化输运理论及自旋电子学的研究 开辟新的领域[29]。

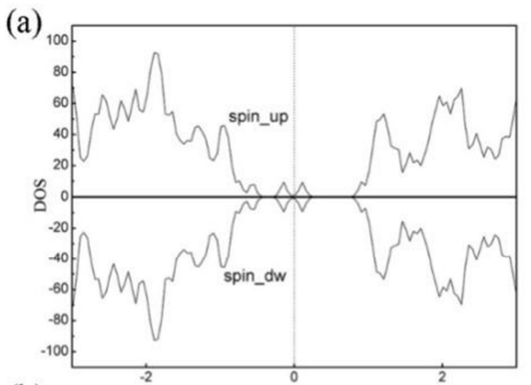

(b)

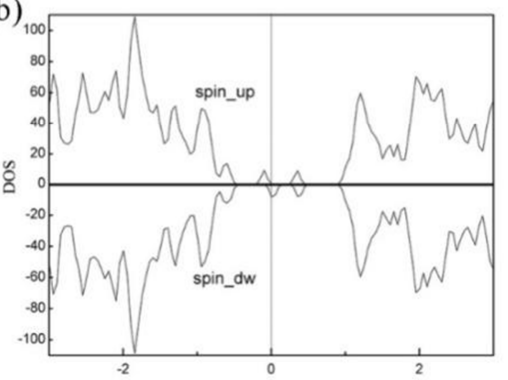

图7 掺杂态密度(a) $\mathrm{V}_{\mathrm{Ge} 2}$ 体系; (b) $\mathrm{V}_{\mathrm{As} 2}$ 体系。 
计算得到 $V_{\mathrm{As} 2}$ 体系磁矩为 $0.4 \mu_{\mathrm{B}}$ 。为了更直观地理解 $\mathrm{V}_{\mathrm{As} 2}$ 体系磁矩的来源, 图8给出了给出了 $\mathrm{V}_{\mathrm{As} 2}$ 掺杂体系的自 旋密度图, 其中等高面值为 $0.001 \mathrm{eV} / \AA^{3}$ 。从图中可以看出, 原子及缺陷附近空间均产生了磁矩。产生磁矩的主要原子 从左到右依次对应为As6, $\mathrm{As} 1, \mathrm{Ge} 2, \mathrm{Ge} 3, \mathrm{As} 3$, 在图8(a) 中用 $\oplus$ 标出。

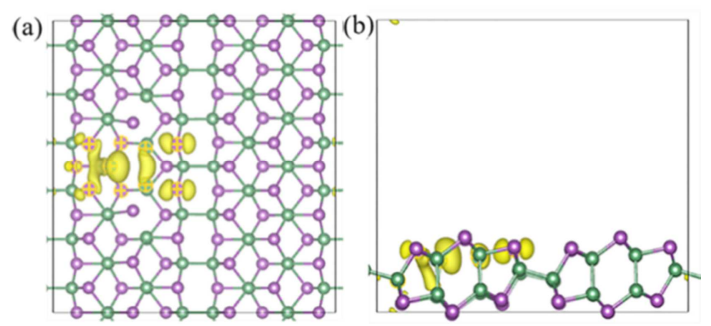

图8 $\mathrm{V}_{\mathrm{As} 2}$ 掺杂体系自旋密度(a)俯视图; (b)侧视图。

表3为空位缺陷附近原子磁矩。表中可以看出各原子 磁矩相加结果小于总磁矩, 说明原子附近空间也产生了磁 矩, 与自旋态密度图体现的结果相同。

表3 $\mathrm{V}_{\mathrm{As} 2}$ 掺杂体系原子磁矩。

\begin{tabular}{llllll}
\hline 原子种类 & As6 & As1 & Ge2 & Ge3 & As3 \\
\hline 磁矩 $/ \mu_{\mathrm{B}}$ & 0.011 & 0.020 & 0.085 & 0.016 & 0.035 \\
\hline
\end{tabular}

\section{4. 结论}

本文利用第一性原理方法, 研究了本征及含空位掺杂 的单层GeAs的几何结构、电学和磁学性质。计算表明本 征单层 $\mathrm{GeAs}$ 是一种禁带宽度为 $1.48 \mathrm{eV}$ 的直接带隙半导体 且无磁性。对单层GeAs材料做空位缺陷掺杂时, 计算得 到在可形成的六种空位掺杂体系中 $\mathrm{V}_{\mathrm{Ge} 2}$ 体系和 $\mathrm{V}_{\mathrm{As} 2}$ 体系相 对更加稳定。 $V_{\mathrm{Ge} 2}$ 和 $\mathrm{V}_{\mathrm{As} 2}$ 均不会使体系引起太大的晶格形 变。 $\mathrm{V}_{\mathrm{Ge} 2}$ 使体系在禁带中产生杂质能级使其能带宽度减小 为 $0.17 \mathrm{eV}$, 但仍为一种直接带隙半导体且无磁性; $V_{\mathrm{As} 2}$ 使 体系产生 $0.4 \mu_{\mathrm{B}}$ 的磁矩, 磁矩由缺陷附近原子及缺陷附近 空间产生, 且自旋向上轨道的非金属性和自旋向下轨道的 金属性致使材料体现半金属性能, 使其可以在自旋电子器 件方面发挥重要作用。

\section{致谢}

本文为国家自然科学基金重点项目《基于忆阻器的存 储计算融合机理与体系结构》(61832007)和国家重点研发 计划资助项目 《 TB级持久性内存存储技术与系统》 (2018YFB1003304)的阶段成果之一。

\section{参考文献}

[1] F. Bonaccorso, L. Colombo, G. Yu, et al., "2D materials. Graphene, related two-dimensional crystals, and hybrid systems for energy conversion and storage," Science, 2015, vol. 347, pp. 1246501.
[2] Z. Ni, Q. Liu, K. Tang, et al., "Tunable bandgap in silicene and germanene," Nano letters, 2012, vol. 12, pp. 113-118.

[3] A. Kara, H. Enriquez, A. P. Seitsonen, et al., "A review on silicene - New candidate for electronics," Surface Science Reports, 2012, vol. 67, pp. 1-18.

[4] A. Fleurence, R. Friedlein, T. Ozaki, et al., "Experimental Evidence for Epitaxial Silicene on Diboride Thin Films," Physical Review Letters, 2012, vol. 108.

[5] P. Vogt, P. De Padova, C. Quaresima, et al., "Silicene: Compelling Experimental Evidence for Graphenelike Two-Dimensional Silicon," Physical Review Letters, 2012, vol. 108.

[6] M. E. Davila, L. Xian, S. Cahangirov, et al., "Germanene: a novel two-dimensional germanium allotrope akin to graphene and silicene," New Journal of Physics, 2014, vol. 16.

[7] F. F. Zhu, W. J. Chen, Y. Xu, et al., "Epitaxial growth of two-dimensional stanene," Nature Materials, 2015, vol. 14.

[8] M. Huang, M. Wang, C. Chen, et al., "Broadband Black Phosphorus Photodetectors with High Responsivity," Advanced materials, 2016, vol. 28, pp. 3481-3485.

[9] J. Qiao, X. Kong, Z. X. Hu, et al., "High-mobility transport anisotropy and linear dichroism in few-layer black phosphorus," Nature communications, 2014, vol. 5, pp. 4475.

[10] C. Barreteau, B. Michon, C. Besnard, et al., "High-pressure melt growth and transport properties of SiP, SiAs, GeP, and GeAs 2D layered semiconductors," Journal of Crystal Growth, 2016, vol. 443, pp. 75-80.

[11] S. Zhang, S. Guo, Y. Huang, et al., "Two-dimensional SiP: an unexplored direct band-gap semiconductor," 2D Materials, 2016, vol. 4, pp. 015030.

[12] P. Wu, and M. Huang, "Stability, bonding, and electronic properties of silicon and germanium arsenides," physica status solidi (b), 2016, vol. 253, pp. 862-867.

[13] Liqin Zhou, Yu Guo, and J. Zhao, "Ge As and Si As monolayers:Novel 2D semiconductors with suitable band structures," Physica E, 2017.

[14] L. Li, W. Wang, P. Gong, et al., "2D GeP: An Unexploited Low-Symmetry Semiconductor with Strong In-Plane Anisotropy," Advanced materials, 2018, vol. 30, pp. e1706771.

[15] B. Mortazavi, and T. Rabczuk, "Anisotropic mechanical properties and strain tuneable band-gap in single-layer SiP, SiAs, GeP and GeAs," Physica E: Low-dimensional Systems and Nanostructures, 2018, pp. S1386947718304387-.

[16] C. S. Jung, D. Kim, S. Cha, et al., "Two-dimensional GeAs with a visible range band gap," Journal of Materials Chemistry A, 2018, vol. 6, pp. 9089-9098.

[17] S. Yang, Y. Yang, M. Wu, et al., "Highly In-Plane Optical and Electrical Anisotropy of 2D Germanium Arsenide," Advanced Functional Materials, 2018, vol. 28, pp. 1707379.

[18] J. Guo, Y. Liu, Y. Ma, et al., "Few-Layer GeAs Field-Effect Transistors and Infrared Photodetectors," Advanced materials, 2018, vol. 30 . 
[19] F. Dazhi, L. Guili, and Z. Shuang, "Effects of vacancy and deformation on an $\mathrm{Al}$ atom adsorbed on graphene," Chinese Journal of Physics, 2018, vol. 56, pp. 689-695.

[20] J. Dai, and J. Yuan, "Modulating the electronic and magnetic structures of P-doped graphene by molecule doping," Journal of physics Condensed matter : an Institute of Physics journal, 2010, vol. 22, pp. 225501

[21] J. Dai, J. Yuan, and P. Giannozzi, "Gas adsorption on graphene doped with B, N, Al, and S: A theoretical study," Applied Physics Letters, 2009, vol. 95, pp. 232105.

[22] P. Giannozzi, S. Baroni, N. Bonini, et al., "QUANTUM ESPRESSO: a modular and open-source software project for quantum simulations of materials," J Phys-Condens Mat, 2009, vol. 21.

[23] P. Giannozzi, O. Andreussi, T. Brumme, et al., "Advanced capabilities for materials modelling with QUANTUM ESPRESSO," J Phys-Condens Mat, 2017, vol. 29.

[24] J. P. Perdew, K. Burke, and M. J. P. R. L. Ernzerhof, "Generalized Gradient Approximation Made Simple," 1998, vol. 77, pp. 3865-3868.
[25] J. P. Perdew, A. Ruzsinszky, G. I. Csonka, et al., "Restoring the Density-Gradient Expansion for Exchange in Solids and Surfaces (vol 100, art no 136406, 2008)," Physical Review Letters, 2009, vol. 102.

[26] D. J. Chadi, "Special points for Brillouin-zone integrations," Physical Review B, 1977, vol. 16, pp. 1746-1747.

[27] A. Q. Cheng, Z. He, J. Zhao, et al., "Monolayered Silicon and Germanium Monopnictide Semiconductors: Excellent Stability, High Absorbance, and Strain Engineering of Electronic Properties," ACS applied materials \& interfaces, 2018, vol. 10, pp. 5133-5139.

[28] X. C. Sun, J. F. Liu, L. C. Kimerling, et al., "Room-temperature direct bandgap electroluminesence from Ge-on-Si light-emitting diodes," Opt Lett, 2009, vol. 34, pp. 1198-1200.

[29] M. N. Baibich, J. M. Broto, A. Fert, et al., "Giant magnetoresistance of $(001) \mathrm{Fe} /(001) \mathrm{Cr}$ magnetic superlattices," 1988, vol. 61, pp. 2472. 